\title{
A Comparative Analysis of Primary Stenting and Optimal Balloon Coronary Angioplasty in Acute Myocardial Infarction. Six Month Results from the STENT PAMI Trial
}

\author{
Luiz Alberto Mattos, Cindy L. Grines, David Cox, J. Eduardo Sousa, Costantino Costantini, Gregg \\ Stone, Marie C. Morice, William O’Neill, Eulógio Garcia, Judith Boura em nome dos investigadores \\ do estudo STENT PAMI
}

São Paulo, SP - Brazil

Objective - To compare the outcome of balloon PT$C A$ with final coronary stenosis diameter $(S D) \leq 30 \%$, with elective coronary stenting.

Methods - We performed a comparative analysis of the 6 month outcomes in patients treated with primary stenting and those who obtained an optimal balloon PT$C A$ result treated during the first 12 hours of AMI onset included in the STENT PAMI randomized trial.

Results - The results were analysed into 3 groups: primary stenting (441 patients, $S D=22 \pm 6 \%$ ), optimal PTCA (245 patients), and nonoptimal $P T C A$ (182 patients, $S D=$ $37 \pm 5 \%)$. At the end of the 6 months primary stent group presented with the lowest restenosis ( 23 vs. 31 vs. $45 \%, p=0.001$, respectively). Ischemia-driven target vessel revascularization rate (TVR) (7vs. 15.5 vs. 19\%, $p=0.001$, respectively).

Conclusion -At the 6 month follow-up, primary stenting offered the lowest restenosis and ischemia-driven TVR rates. Compared to optimal balloon PTCA. Nonoptimal primary balloon PTCA pts (SD=31-50\%), had the worst late angiographic outcomes and should be treated more actively with coronary stent implantation.

Key words: stent, angioplasty, restenosis

From the division of Interventional Cardiology of the Instituto Dante Pazzanese de Cardiologia, São Paulo, Brazil (LAM e JES); William Beaumont Hospital, Royal Oak, MI, USA (CLG,WON e JB); Presbyterian Hospital, Charlotte, NC, USA (DC); Clínica Cardiológica Costantino Costantini, Curitiba, Brazil (CC); Washington Hospital Center, Washington, DC, USA (GWS); L'Institut Cardiovasculaire Paris Sud, Antony, France (MCM) e Hospital Gregório Maranon, Madrid, Spain (EG).

Mailing address: Luiz Alberto Mattos - Av. Jandira, 550/121 - 04080-003 - São Paulo, SP - E-mail: pivmattos@uol.com.br

Funding provided by Cordis, at the Johnson \& Johnson Company, Warren, New Jersey, USA. Other members of the STENT PAMI study group are listed in the Appendix. The results were partially presented at the 48th Sessions of the American College of Cardiology Meeting, March 1999, New Orleans, LA, USA.
A series of randomized trials were performed to obtain a comparative analysis between two percutaneous revascularization methods, primary coronary stent implantation and primary balloon PTCA ${ }^{1-3}$, available for the treatment of acute myocardial infarction (AMI) in the first 12 hours of its onset. The STENT PAMI trial randomized a greater number of AMI patients than did the previous trials (900) in 62 centers worldwide, concluding that primary heparin-coated Palmaz-Schatz stent implantation offered greater final luminal diameter, a lower restenosis rate and fewer ischemia-driven target vessel revascularization (TVR) procedures at the end of the first 180 days when compared with balloon PTCA ${ }^{4}$.

The BENESTENT I trial ${ }^{5}$ compared the elective coronary stent implantation strategy with balloon PTCA in non-AMI patients showing that $36 \%$ of patients randomized to the balloon obtained a final procedural stenosis diameter lower than $30 \%$, and with reduced restenosis rates with fewer occurrences of major coronary events at the long-term follow-up, benefits similar to those obtained with routine stenting. Serruys et al referred to these optimal balloon PTCA results as a stent-like procedure ${ }^{6,7}$. The clinical and cost-effectiveness implications of these findings have stimulated the specific design of trials capable of proving or disproving this conclusions.

In the first 12 hours of AMI onset, this comparison was not yet realized. The objective of this study was to perform the comparative analysis of the clinical and angiographic results at the end of the first 6 months after AMI of patients who had been submmited to a routine coronary stent implantation and those who obtained an optimal primary balloon PTCA angiographic result, and were included in the STENTPAMI trial.

\section{Methods}

This analysis follows the protocol determined for the 
main STENT PAMI trial. We included patients of both sexes, above 18 years of age, within the first $12 \mathrm{~h}$ of AMI (symptions and ST-T segment elevation $\geq 1 \mathrm{~mm}$ in more than 2 contiguous ECG leads). Clinical exclusion criteria included prior administration of thrombolytics for the index infarction, current use of warfarin, stroke within one month, renal failure, cardiogenic shock, expected survival of < 1 year, women of childbearing potential unless a recent pregnancy test was negative, or known contraindications to aspirin, heparin, or ticlopidine.

The patients were medicated at the emergency room with aspirin (325mg/PO), ticlopidine $(500 \mathrm{mg} / \mathrm{PO}$, for 30 days), heparin (5,000 to 10,000IU/IV), beta blockers (IV) in the absence of contraindications.

The coronary arteriography and left ventriculography were performed with the Judkins technique with low osmolar ionic contrast medium. Only the infarct-related artery was treated. Once flow was established (either spontaneously or after initial balloon inflations), the operator determined whether the infarct lesion(s) qualified for randomization. The randomization process was conducted with a central computerized system (Amsterdam, The Netherlands).

The technicians selected only native arteries, $3.0-4.5 \mathrm{~mm}$ reference segment diameter (visual), and lesion(s) that could be covered with one or two stents (15 mm length). Vessels were also excluded if the stent would protrude into the left main artery (ostial LAD or circumflex lesions), if large branches ( $\geq 3.0 \mathrm{~mm}$ in diameter) would be constricted by the stent, or if tortuosity and calcification made it unlikely that the stent could be delivered and expanded.

Patients randomized for routine coronary stent implantation and received a $15-\mathrm{mm}$ Palmaz-Schatz heparincoated stent mounted on a balloon that incorporated a sleeved stent delivery system. After stent deployment at $8 \mathrm{~atm}$, a separate high pressure inflation of $\geq 16$ atm was recommended. The objective was the achievement of an optimal stent implantation, with an SD $\leq 10 \%$ (visual assessment), without edge dissections or severe adjacent nontreated stenosis.

In patients randomized to ballon PTCA, the technique already tested was used, with the goal of a final stenosis as low as possible. At the operators discretion, coronary stents could be implanted in the presence of suboptimal results (stenosis diameter $\geq 50 \%$ or flow threatening dissections), using other commercially available stent.

All the procedures were performed with full intravenous heparinization (ACT $\geq 350$ seconds). Administration of thrombolytics or abciximab was discouraged. Intracoronary ultrasound was not mandatory, being performed at the technician's discretion.

Clinical and angiographic analysis - The cumulative occurrence of all major clinical events was monitored throughout the index hospitalization, and at 1 and 6 months. Prior to performance of the protocol follow-up angiography at 6.5 months, investigators documented the angina class or evidence of ischemia at stress testing.

Detailed case report forms were completed by clinical coordinators at each site. Independent monitors traveled to the sites to verify source documentation on all patients. All acute and follow-up cineangiograms were obtained using standard acquisition guidelines and submitted to independent Angiographic Core Laboratories (Washington Hospital Center, Washington D.C. and Cardialysis, Rotterdam, The Netherlands). The quantitative coronary angiographic (QCA) analysis was performed using the CAAS II system (Pie Medical, The Netherlands). Myocardial perfusion was graded using the Thrombolysis in Myocardial Infarction (TIMI) classification. The mean reference diameter and the minimal lumen diameter were used to calculate the percent $\mathrm{SD}$. An MLD = 0.0 was assumed in the presence of a total occlusion at baseline or follow-up.

The primary objective was to assess the combined cumulative occurrence of death, reinfarction, stroke, or ischemia-driven TVR procedures at the end of the first 6 months after the index AMI. Each component of the primary endpoint was adjudicated by an independent clinical events committee.

Secondary endpoints were the 6-month minimal lumen diameter and $\mathrm{SD}$, restenosis rate $(\geq 50 \% \mathrm{SD})$, and reocclusion (TIMI 0-1 flow in a vessel that was previously patent) ${ }^{8}$.

In this current analysis, the randomized patients were divided into 3 different groups according to the final procedural SD, after QCA analysis: routine stenting, optimal PTCA (SD $\leq 30 \%)$, and nonoptimal PTCA (SD=31-50\%).

Statistical analysis - Categorical variables were presented as frequency and percentual, and continuous variables as mean an standard deviation.

Categorial variables were analysed with $\mathrm{Bi}$-caudal ChiSaqure Test or Exact Fischer test, whichever fitted better. Continuous variables were analysed by linear regression model taking the subset (stent, optimal PTCA and non-optimal PTCA) as independent variable. A later analyse was done to compare pairs of subsets.

\section{Results}

Between December 1996 and December 1997, 900 patients were randomized. From these, the cinefilms of 868 (96\%) were analyzed by the Central Angiographic core labs, with the calculation of the final procedural stenosis diameter by QCA.

According to the prespecified criteria, patients were divided into 3 groups: routine stenting, 441 (51\%) patients; optimal balloon PTCA, 245 (28\%); and nonoptimal balloon PTCA, $182(21 \%)$ patients.

The clinical profile of these groups are displayed in table I. The clinical variables were well balanced among all 3 groups, without any statistical significance. The mean age was 60 years, more than $70 \%$ of participants were men and $15 \%$ had diabetes. The mean time between pain onset and emergency room admission was 160 minutes.

The angiographic profile is shown in table II, also without any major differences among the 3 groups. The majority of the patients had anterior myocardial infarction related to LAD occlusions, and more than $45 \%$ of the group 


\begin{tabular}{|lccc|}
\hline \multicolumn{4}{|c|}{ Table I - Clinical characteristics of the 3 groups analyzed } \\
\hline \multirow{2}{*}{$\%$ of patients } & Routine & Optimal & Nonoptimal \\
& stenting & PTCA & PTCA \\
& $\mathrm{N}=441$ & $\mathrm{~N}=245$ & $\mathrm{~N}=182$ \\
\hline Mean age (years) & $61 \pm 12$ & $59 \pm 12$ & $60 \pm 13$ \\
Men & 75 & 74 & 77 \\
Diabetics & 15 & 16 & 14 \\
Hypertensives & 42 & 41 & 43 \\
Previous events & 10 & 13 & 9 \\
AMI & 1 & 2 & 2 \\
CABG & & & \\
\hline p= NS. & & & \\
\hline
\end{tabular}

exhibited multivessel coronary heart disease. The mean global ejection fraction prior to the intervention was greater than $45 \%$.

The optimal balloon PTCA patients required bail-out stent implantation in $23 \%$ of the group. On the contrary, only $3 \%(p<0.05)$ of nonoptimal PTCA patients received nonplanned stents. Regarding the number of stents required per artery, the average was similar between the routine stenting group and the optimal PTCA (1.4) group, but we observed a trend toward a higher number of stents per artery in the nonoptimal PTCA group (2.2).

The acute procedural results are demonstrated in table III. The coronary TIMI flow grade 3 was re-established in $90 \%$ of routine stent patients, $93.5 \%$ of the optimal PTCA group, and $92 \%$ of the nonoptimal PTCA patients ( $\mathrm{p}=\mathrm{NS}$ ), assessed by core lab analysis.

The acute procedural QCA results revealed differences among the groups. The mean reference artery diameter was lower for the optimal balloon PTCA group. The greater ML$D$ was obtained in the routine stent group and follows a lower progressive sequence, for the optimal and nonoptimal PTCA groups (2.6 \pm 0.4 vs. $2.3 \pm 0.4$ vs. $1.9 \pm 0.4 \mathrm{~mm}$, respectively, $\mathrm{p}<0.05)$. The higher acute gain with the lower stenosis diameterfollowed that same sequence for the 3 different groups, all statistical differences favoring the routine stent patients (table III).

At the end of the first 6 months after AMI, 669 (77\%) of the patients underwent a new protocol cineangiography, with a similar percentage for all groups $(77,78$, and $75 \%$, respectively, $\mathrm{p}=\mathrm{NS}$ ). The greater MLD at the follow-up was

\begin{tabular}{|lccc|}
\hline \multicolumn{4}{|c|}{ Table II - Angiographic profile of the 3 groups } \\
\hline \% of patients & Routine & Optimal & Nonoptimal \\
& stenting & PTCA & PTCA \\
& $\mathrm{N}=441$ & $\mathrm{~N}=245$ & $\mathrm{~N}=182$ \\
\hline & & & \\
& 40 & 45 & 42 \\
Vessel treated & 14 & 12 & 16 \\
Left anterior descending & 45 & 43 & 42 \\
Left circumflex & 46 & 43 & 46 \\
Right coronary & $49 \pm 12$ & $48 \pm 11$ & $48 \pm 11$ \\
Multivessel disease & $1.4 \pm 0.6$ & $1.4 \pm 0.6$ & $2.2 \pm 0.8$ \\
Mean ejection fraction & \multicolumn{3}{c}{} \\
\# stents/vessel & \multicolumn{3}{c}{} \\
\hline p= NS. &
\end{tabular}

\begin{tabular}{|c|c|c|c|c|}
\hline \multicolumn{5}{|c|}{$\begin{array}{l}\text { Table III - Acute procedural results and quantitative angiographic } \\
\text { analysis of the } 3 \text { groups }\end{array}$} \\
\hline$\%$ of patients & $\begin{array}{l}\text { Routine } \\
\text { stenting }\end{array}$ & Optimal & $\begin{array}{l}\text { Nonoptimal } \\
\text { PTCA }\end{array}$ & $\mathrm{p}$ \\
\hline & $\mathrm{N}=441$ & $\mathrm{~N}=245$ & $\mathrm{~N}=182$ & \\
\hline TIMI flow - 1 & 0.4 & 0.4 & 0 & \\
\hline-2 & 9.6 & 6.1 & 8.2 & \\
\hline-3 & 90 & 93.5 & 92 & 0.6 \\
\hline Nonplanned stents & - & 23 & 3 & 0.0001 \\
\hline Residual dissection & 6 & 18 & 28 & 0.0001 \\
\hline Final pressure (ATM) & $15 \pm 4$ & $9 \pm 4$ & $9 \pm 3$ & 0.001 \\
\hline Balloon/artery ratio & $1.1 \pm 0.2$ & $1.2 \pm 0.1$ & $1.1 \pm 0,2$ & 0.7 \\
\hline $\begin{array}{l}\text { Final coronary DS } \\
(\mathrm{mm})\end{array}$ & $18 \pm 6$ & $22 \pm 6$ & $37 \pm 5$ & 0.0001 \\
\hline Reference diameter & $3.2 \pm 0.4$ & $2.9 \pm 0.4$ & $3.1 \pm 0.5$ & 0.001 \\
\hline MLD preprocedure & $0.4 \pm 0.5$ & $0.4 \pm 0.5$ & $0.4 \pm 0.5$ & 0.7 \\
\hline Postprocedure & $2.6 \pm 0.4$ & $2.3 \pm 0.4$ & $1.9 \pm 0.4$ & 0.001 \\
\hline Acute gain & $2.2 \pm 0.6$ & $1.9 \pm 0.7$ & $1.5 \pm 0.6$ & 0.001 \\
\hline
\end{tabular}

observed in the routine stenting group, becoming lower for the optimal and nonoptimal balloon PTCA groups $(1.8 \pm 0.7$ vs. $1.7 \pm 0.7$ vs. $1.5 \pm 0.8 \mathrm{~mm}$, respectively, $\mathrm{p}<0.05)$. Lower stenosis was also observed in the same sequence of groups ( 40 vs. 44 vs. $53 \%$, respectively, p<0.05).

The restenosis rate was lower for the patients included in the routine stenting group (table IV), even including the comparison with the optimal balloon PTCA group (23 vs. 31 vs. $45 \%$, respectively, $p=0.001$ ). In relation to the occurrence of the reocclusion phenomenon, patients included in the routine stenting group or with optimal PTCA results did not demonstrate significant differences (5 vs. $6 \%, \mathrm{p}=\mathrm{NS}$ ), showing a benefit when compared with to nonoptimal PTCA patients who had twice that number $(6 \mathrm{vs}$. $13 \%, \mathrm{p}=0.0008$ ).

The primary endpoint events was observed significantly less often in the routine stenting strategy group (table V). The occurrence of combined clinical events was $12 \%$ (routine stenting), $17.5 \%$ (optimal PTCA), and $22.5 \%$ (nonoptimal PTCA), respectively. The clinical benefit was only detected in the comparison of routine stenting patients with optimal balloon PTCA patients $(p=0.001)$. In the side-by-

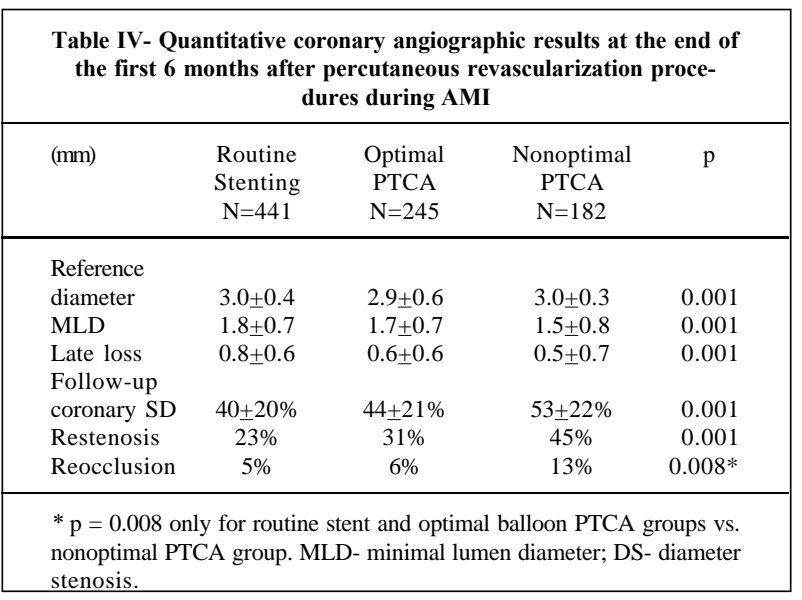




\begin{tabular}{|c|c|c|c|c|}
\hline \multicolumn{5}{|c|}{$\begin{array}{l}\text { Table } \mathrm{V} \text { - Clinical combined events at the end of the first } 6 \text { months } \\
\text { after AMI of the } 3 \text { different procedural results }\end{array}$} \\
\hline$\%$ of patients & $\begin{array}{l}\text { Routine } \\
\text { Stenting } \\
\mathrm{N}=441\end{array}$ & $\begin{array}{c}\text { Optimal } \\
\text { PTCA } \\
\mathrm{N}=245\end{array}$ & $\begin{array}{c}\text { Nonoptimal } \\
\text { PTCA } \\
\text { N=182 }\end{array}$ & $\mathrm{p}$ \\
\hline Death & 4 & 1.2 & 3.3 & 0.2 \\
\hline Reinfarction & 2.4 & 2 & 2.7 & 0.9 \\
\hline Stroke & 0.2 & 0.4 & 0 & 0.8 \\
\hline Ischemia-driven TVR & 7 & 15.5 & 19 & $0.001 *$ \\
\hline Combined events & 12 & 17.5 & 22.5 & $0.003 *$ \\
\hline \multicolumn{5}{|c|}{$\begin{array}{l}\mathrm{p}<0.05 \text { only for routine stenting group vs. optimal and nonoptimal bal- } \\
\text { loon PTCA groups. TVR- target vessel revascularization. }\end{array}$} \\
\hline
\end{tabular}

side comparison of the occurrence of the primary endpoint between optimal balloon and nonoptimal PTCA groups, we did not find significant differences.

The reduction in the ischemia-driven TVR rate (routine stenting, 7 vs. optimal PTCA, $15.5 \%, \mathrm{p}=0.001$ ) was the only clinical variable analyzed that exhibited an expressive benefit when patients participated in a routine stenting strategy. However, this benefit was not observed in the comparison of optimal and non-optimal balloon PTCA groups (15.5 vs. $19 \%$, respectively, $\mathrm{p}=\mathrm{NS}$ ). The significant reduction observed in this clinical variable had a major contribution for a lower rate of clinical combined events.

The major complications related to AMI occurrence (death, reinfarction, and stoke) were not different among the 3 groups. A routine stenting strategy did not modify their occurrence.

\section{Discussion}

In the current analysis, we compared the acute (30 days) and follow-up (6 months) of more than 800 patients randomized in the STENT PAMI trial ${ }^{4}$, during the first 12 hours of AMI onset, according to the final procedural stenosis diameter, assessed by QCA analysis. The group of patients originally randomized to the balloon PTCA strategy were divided according to the achievement of an optimal result (stent-like), w than stenosisdiam eter $\leq 30 \%$, and compared with those randomized to the routine stenting strategy.

The clinical and angiographic results were obtained in a cumulative fashion at the end of the first and sixth months after AMI. The acute results did not reveal significant differences in the success rate, the re-establishment of TIMI 3 flow rate, and the occurrence of major complications. We only observed that patients who underwent a routine stenting strategy had a significantly lower procedural stenosis diameter, greater lumen gain and, as a consequence, a higher MLD, when compared with other groups. The marked differences in the results were observed in the clinical and angiographic analyses at the end of the first 6 months. So, the group of patients who had routine stenting exhibited a significantly lower restenosis rate (23\%) and, as a consequence, a lower need for new TVR ischemia-driven procedures, reduced in more than $50 \%$ of patients. The group classified as having an optimal balloon PTCA result, despite being favored by $23 \%$ over coronary stent implantation, did not obtain similar clinical and angiographic benefits as compared with those who participated in the routine stenting strategy.

The late reocclusion rate was similar for routine stenting and optimal balloon PTCA groups, however $23 \%$ of patients in the optimal ballon PTCA group had stent implant that may have protected them from this hazardous complication, compared with the nonoptimal balloon PTCA patients $(5-6 \%$ vs. $13 \%, \mathrm{p}<0.05)$.

Optimal Balloon PTCA or Routine Stenting Strategy in AMI? - Since the subanalysis from the elective randomized trial BENESTENT I (balloon PTCA vs. coronary stenting), Serruys et al ${ }^{5-7}$ have made the interesting observation that a range of 30 to $40 \%$ of patients who undergo only balloon PTCA who had achieved a final procedural stenosis diameter $\leq 30 \%$, had a favorable long-term outcome similar to that observed in patients randomized to stenting. Because of this, the concept of a stent-like result was created ${ }^{6}$.

The ability of coronary stents to improve balloon PTCA suboptimal results either by sealing dissection planes or reducing the restenosis rate are unquestionable and very well proven ${ }^{5,7}$. However, the multiple morphological presentations of coronary heart disease may draw into question routine stent application, which may not be suitable for all patients for example, those with long and tandem coronary stenosis, small vessels, bifurcations, and the present and possible scenario of in-stent restenosis ${ }^{8}$.

In non-AMI patients, recent studies questioned the concept of optimal balloon PTCA with a stent-like result ${ }^{6}$. All of the studies discussed in their methods sections adjunct assisted devices capable of monitoring on-line the coronary stent implantation procedure. These devices can verify anatomical (IVUS or QCA) or physiological (coronary flow reserve by Doppler catheters) variables ${ }^{9,10}$. The results of the studies DEBATE I ${ }^{11}$, DESTINI ${ }^{12}$ and DEBATE II ${ }^{13}$ were all very similar. When balloon catheters were able to obtain a lower stenosis diameter ( $<35 \%$ by QCA) associated with a normalization of coronary flow reserve $(>2.0)$, the long-term clinical and angiographic results became very similar to those observed with routine stent application. However, a very wide range of patients were able to achieve that optimal result, from 25 to $50 \%$. Additionally, in the DEBATE II trial, $25 \%$ of the patients required bail-out coronary stent implantation, and a surprising finding was that patients who, after obtaining optimal results received a stent by protocol decision, showed even lower rates of new late major clinical events (1\%). These findings associated with the additional costs and longer procedures do not recommend these methods for all patients treated in daily practice.

The report of the most recent trial (OPUS ${ }^{14}$ ), applying a more friendly technology available in all major invasive cardiac laboratories (visual assessment with assisted QCA) has been released. Elective non-AMI patients $(n=500)$ with reference vessels $\geq 3$.0mm and stenosis length $<20 \mathrm{~mm}$ were randomized into either the routine stenting strategy or to the achievement of an optimal balloon PTCA (SD $\leq 30 \%)$. At 
the end of the first 6 months after the index procedure, patients included in the stent arm showed a significant reduction in major combined clinical events (6 vs. $15 \%, \mathrm{p}<0.003)$ and in the ischemia-driven TVR rates ( 4 vs. $11 \%, \mathrm{p}<0.001$ ). These results obtained in non-AMI patients were quite similar to those observed in our patients treated during the first hours of AMI onset.

The application of optimal balloon PTCA during AMI - Until now, no randomized sub-analysis of these two strategies in the setting of AMI has been performed. We proved that patients who underwent routine stenting during AMI exhibited better late clinical and angiographic results. The mechanisms of the restenosis process are complex and multifactorial, involving elastic recoil and excessive intimal hyperplasia ${ }^{13}$. The benefit of coronary stent implantation in the reduction of clinical and angiographic restenosis rates are strongly related to the acute procedural result (lower stenosis diameter) mixed with the positive effect of the chronic elastic recoil phenomenon. These facts were also observed in this AMI analysis.

Patients included in the optimal balloon PTCA group did not achieve the same clinical and angiographic results obtained in patients treated with the routine stenting strategy. Despite a $23 \%$ cross-over rate of stenting in this group, the only significant benefit was the avoidance of late infarct-related vessel reocclusion.

However, the percutaneous strategy of routine stenting during the first 12 hours of AMI did not modify the rates of major complications (death, reinfarction, and stroke) ${ }^{10}$. The achievement of a final procedural stenosis diameter $\leq 50 \%$, classified as nonoptimal balloon PTCA, was enough to equalize the occurrence of these major clinical complications among all 3 groups.

Should we Perform Routine Stenting during AMI? - If our goal during the AMI mechanical reperfusion treatment is a reduction in the rates of major clinical complications (death and reinfarction), routine stenting will not provide us with these achievements ${ }^{15}$. The fast re-acquisition of normal TIMI 3 coronary flow in the infarct-related artery still persists as the main endpoint in the AMI setting, and that is achieved even with an stenosis diameter $\leq 50 \%$, either with stent implantation or balloon PTCA ${ }^{4,10}$.

However, if we want to prevent late restenosis occurrence, even after an optimal balloon PTCA result (SD $\leq 30 \%$ ), we should implant coronary stents. It is important to mention that these findings were observed in patients with total native coronary stenosis, in vessels $\leq 3.0 \mathrm{~mm}$ (visual) and with a lesion length $\leq 30 \mathrm{~mm}$, with good distal run-off, without major bifurcations into large side branches. Until new evidence is available, we cannot promote this recommendation for other angiographic scenarios.

We also demonstrated that the categorization of a primary PTCA procedure as nonoptimal (stenosis diameter $=31$ $50 \%$ ) implies that in $50 \%$ of patients a greater chance exists of suffering a late vessel reocclusion. This undesirable phenomenon occurred in $13 \%$ of these patients. The negative impact on the recovery of left ventricular function has already been well-documented ${ }^{14,15}$,justifying a more liberal stent approach in this situation.
Study Limitations - The patients randomized to balloon PTCA only received stents in the face of a suboptimal angiographic result (stenosis diameter $>50 \%$ with or without severe dissections) as a rescue or bail-out procedure, but not as a provisional stenting strategy.

The IIb/IIIa inhibitors were not widely used in this trial ( $<5 \%$ ), according to the protocol requirement. Recent randomized studies ${ }^{16,17}$ have demonstrated an optimization of the acute and late follow-up results, with the association of these new pharmacological agents, either when balloon or stent were applied. New ongoing trials ${ }^{18-21}$ may clarify this issue.

Conclusions - In a selected group of AMI patients who underwent mechanical reperfusion techniques, the routine stenting strategy promoted a significant reduction in the final procedural stenosis diameter, with a $50 \%$ reduction in the 6 month restenosis and ischemia-driven TVR rates, when compared with patients who achieved an optimal balloon PTCA result (stenosis diameter $\leq 30 \%$ by QCA).

The group of patients classified as having nonoptimal balloon PTCA results ( stenosis diameter $=331-50 \%$ ) obtained the worst clinical and angiographic results with twice the late reocclusion rate, as compared with the other 2 groups. Coronary stent implantation was used in few patients (3\%), justifying in the face of a final stenosis diameter $>30 \%$, a more liberal stent approach.

Appendix

Steering Committee Members

Cindy Grines (Chairperson), Royal Oak, MI, USA;

Marie-Claude Morice, (Coordinator of European, Asia and Middle East Centers), Antony, France; Luiz Alberto Mattos (Coordinator of South America Centers), Sao Paulo, Brazil;

Gregg Stone, Washington, DC, USA; William O’Neill, Royal Oak, MI, USA; Olivier Madona, Paris, France; Brian Firth, Warren, NJ, USA; and Gerrit Van-Ness, Rotterdam, The Netherlands.

\section{Data Base Coordinator Centers:}

Marcel Eijgleshoven, Rotterdam (Cardialysis), The Netherlands and Mariann Graham, Royal Oak (William Beaumont Hospital), MI, USA.

Central Adjudication Committee (Clinical Events)

Thomas Ryan (Chairman), Boston, MA, USA; Felix Zijlstra, Zwolle, The Netherlands; Patrick Serruys, Rotterdam, The Netherlands; Spencer King, Atlanta, GA, USA, and Richard Kuntz, Boston, MA, USA.

Central Result Coordinator

Cardialysis, Rotterdam, The Netherlands.

\section{Central Clinical Events Committee}

William Winjs (Chairman), Aalst, Belgium and Martin Kutryk, Rotterdam, The Netherlands.

Central Core Angiographic Laboratories:

Jeffrey Popma and Alexandra Lansky, Washington 
Hospital Center, DC, USA, and Marcel Van den Brand, Cardialysis, Rotterdam, The Netherlands.

\section{Principal Investigators and Hospital Sites North America:}

D. Cox, Presbyterian Hospital, Charlotte, NC; C. Grines, William Beaumont, Royal Oak, MI; S. Katz, North Shore University Hospital, Manhasset, NY; A. Giambartolomei, St. Joseph's Hospital. Syracuse, NY; B. Brosdie, LeBauer Health Center, Greensboro, NC; B. Donohue, Allegany General Hospital, Pittisburg, PA; J. Griffin, Virginia Beach Hospital, Virginia Beach, VA; P. Casele, Lancaster Heart Foundation, Lancaster, PA;F.Feit, New YorkUniversity MedicalCenter,New York, NY; M. Ayres, Horizon Physicians Group, Knoxville, TN; E. Kosinsky, St. Vicent's (@ check the spelling. Should it be Vincent's?) Medical Center, Bridgeport, CT; D. Roberts, Sutter Memorial, Sacramento, CA; T. Hanlon, St. Charles Hospital, Bend, OR;F.St. Gore, ElCamino Hospital, Mountain View,CA; J. Hartmann,. Midwest Heart Research Foundation, Lombard, IL;D. Rizik, Arizona Heart Institute-Osborne, Sccottsdale, AZ; K. Ford, Western Baptist Hospital, Paducah, KY; J. Werner, Overlake Hospital Medical Center, Bellevue, WA; J. Martin, BrynMawrHospital, Bryn Mawr,PA; R. Smalling, StJohn's Regional Health Center, Springfield, MA; P. Kraft, Henry Ford Hospital, Detroit, MI; T. Linnemeier, Indiana Heart Institute, Indianapolis, IN; P. Overlie, Methodist Heart Center, Lubbock, TX; I. Penn, Vancouver Hospital Center, Vancouver, Canada; S. West, Lakeview Hospital, Bountifiul, UT; T. Feldman, University of Chicago, Chicago, IL; C. Cates, The Atlanta Cardiology Group, Atlanta, GA; N. Kander, Riverside Hospital, Columbus, OH; J. Assala, Barnes Hospital, St. Louis, MO; M. Leon, Washington, DC; R. Heuser, Columbia Medical Center, Phoenix, AZ;D. Williams, Rhode Island Hospital, Providence, RI; and J. Burke, Temple Cardiology, Philadelphia, PA.

\section{Europe}

E. Garcia, Hospital Gregorio Maranon, Madrid, Spain, F. Kiemeney, Onze Lieve Gasthuis, Amsterdam, The Netherlands; M. Morice, L'Institut Cardiovasculaire Paris Sud, Antony, France; M. Piper, Herz Zentrum Bodensee, Kreuzlingen, Switzerland; C. Macaya, Hospital Clínico San Carlos, Madrid, Spain; G. Binaghi, Ospedale di Cirolo, Varese, Italy; W. van der Giessen, Thoraxcenter, Rotterdam, The Netherlands; W. Rutsch, Klin. Charite der Humbolt University, Berlin, Germany; P. Heuvel, Academisch Zickenhuis Middelheim, Antuerp, Belgium; T. Lefevre, Institut Cardiovasculaire Paris Sud, Antony, France; C. Loubeyre, Institut Cardiovasculaire Paris Sud, Quincy-Sous-Senart, France; M. Vandormael, Clinique Saint Jean, Brussels, Belgium; P. Materne, Hospital de la Citadelle, Liege, Belgium; J. Marco, Clinique Pasteur, Toulouse, France; P. Probst, Universitastklinik fur, Vienna, Austria; A. Bartorelli, Universidade of Milan, Milan, Italy; F. Fernandez-Aviles, Hospital Universitário de Valladolid, Valladolid, Spain; B. Glatt, Centre Cardiologique de Nord, Saint Denis, France; J Goy, Centre Hospital Universitaire Vaudois, Lausanne, Switzerland; and C. Hamm, University Hospital Eppendorf, Hamburg, Germany.

\section{South America}

J. Eduardo Sousa, Inst. Dante Pazzanese de Cardiologia, Sao Paulo, Brazil; C. Costantini, Santa Casa de Misericórdia, Curitiba, Brazil; A. Sampaolessi, Inst. Modelode Cardiologia, Cordoba, Argentina; C. Vozzi, Sanatório Los Arroyos, Rosario, Argentina; J. Belardi, Inst. Cardiovascular de Cardiologia, Buenos Aires, Argentina; C. Conti, Inst. Di Cardiologia, Buenos Aires, Argentina; and L. Grinfeld, Hospital Italiano de Buenos Aires, Buenos Aires, Argentina.

\section{Middle East and Asia}

K. Niazi, Faisal Specialist Hospital, Ryadh, Saudi Arabia and M. Nobuyoshi, Kokura Memorial Hospital, Kitakyushu, Japan.

\section{References}

1. Antoniucci D, Santoro G, Bolognese L, et al. A clinical trial comparing primary stenting of the infarct-related artery with optimal primary angioplasty for acute myocardial infarction. Results form the Florence Randomized Elective Stenting in Acute Coronary Occlusions (FRESCO) trial. J Am Coll Cardiol 1998; 31: 1234-9.

2. Suryapranata H, Vant Hoff AWJ, Hoorntje JCA, et al. Randomized comparison of coronary stenting with balloon angioplasty in selected patients with acute myocardial infarction. Circulation 1998; 97: 2502-05.

3. Rodriguez A, Bernardi V, Fernandez M, et al. In-hospital and late results of coronary stents versus conventional balloon angioplasty in acute myocardial infarction (GRAMI trial). Am J Cardiol 1998; 81: 1286-91.

4. Grines C, Cox D, Stone GW, et al. Coronary angioplasty with or without stent implantation for acute myocardial infarction. N Engl J Med 1999; 341: 1949-56.

5. Serruys PW, de Jaegere P, Kiemeney F, et al. A comparison of balloon expandable stent implantation with balloon angioplasty in patients with coronary heart disease. N Engl J Med 1994; 331: 489-95.

6. Serruys PW, Azar AJ, Sigwart U, et al. Long-term Follow up of "Stent-like" ( $\leq 30 \%$ Diameter Stenosis Post) Angioplasty: A case for provisional stenting. J Am Coll Cardiol 1996; 27: 15 A.
7. Serruys PW, Kay IP, Disco C, et al. Periprocedural quantitative coronary angiography after Palmaz-Schatz stent implantation predicts the restenosis rate at six months. Results of a meta-analysis of the Belgian Netherlands Study (BENESTENT) I, BENESTENT II Pilot, BENESTENT II and MUSIC Trials. J Am Coll Cardiol 1999; 34: 1067-74.

8. The TIMI Study Group. The Thrombolysis in myocardial infarction (TIMI) trial: Phase I findings. N Engl J Med 1985; 312: 932-6.

9. Stone GW. The CADILLAC Study: In-hospital results. 72nd Scientific Sessions of the American Heart Association Meeting. Atlanta, GA, USA, November 8, 1999.

10. Grines CL, Cox D, Stone GW, et al. STENT PAMI: 12 months results and predictors of mortality. J Am Coll Cardiol 2000; 35: 402 A.

11. Serruys PW, Di Mario C, Piek J, et al. Prognostic value of intracoronary flow velocity and diameter stenosis in assessing the short- and long-term outcomes of coronary balloon angioplasty: the DEBATE study (Doppler Endpoints Balloon Angioplasty Trial Europe). Circulation 1997; 96: 3369-77.

12. Di Mario C, Moses J, Muramatusu T, et al. Multicenter randomized comparison of primary stenting vs. balloon angioplasty optimized by QCA and intracoronary Doppler: procedural results in 580 patients. Eur Heart J 1998; 19: 567 A.

13. Serruys PW, de Bruyne B, de Sousa JE, et al. DEBATE II: a randomized study to 
evaluate the need of additional stenting after guided balloon angioplasty. Eur Heart J 1998; 19: 567 A.

14. Ferguson J. Meeting Highlights: 48th Annual Scientific Sessions of the American College of Cardiology. Circulation 1999; 100: 573.

15. Montalescot G. The ADMIRAL Study: abciximab with PTCA and Stent in AMI. 48th Annual Scientific Session of the American College of Cardiology, New Orleans, LO, USA, March 7, 1999.

16. Grines CL, Brodie B, Griffin J, et al. Which primary PTCA patients may benefit form new technologies? Circulation 1995; 92: I-146.

17. Lincoff AM, Califf RM, Moliterno DJ, et al. Complementary clinical benefits of coronary-artery stenting and blockade of platelet glycoprotein IIb/IIIa receptors. N Engl J Med 1999; 341: 319-27.

18. Topol EJ, Mark D, Lincoff AM, et al. Outcomes at 1 year and economic implications of platelet glycoprotein IIb/IIIa blockade in patients undergoing coronary stenting: results from a multicenter randomised trial. Lancet 1999; 354: 2019-24.

19. Brener SJ, Barr LA, Burchenal MD, et al. Randomized, placebo-controlled trial of platelet glycoprotein IIb/IIIa blockade with primay angioplasty for acute myocardial infarction. Circulation 1998; 98: 734-41.

20. Neumann FJ, Blasini R, Schmitt C, et al. Effect of glycoprotein IIb/IIIa receptor blockade on recovery of coronary flow and left ventriculat function after the placement of coronary-artery stents in acute myocardial infarction. Circulation 1998; 98: 2695-01.

21. Neumann FJ, Kastrati A, Schmitt C, et al. Effect of glycoprotein IIb/IIIa receptor blockade with abciximab on clinical and angiographic restenosis rate after the placement of coronary stents following acute myocardial infarction. J Am Coll Cardiol 2000; 35: 915-21. 